\title{
NR. 14
}

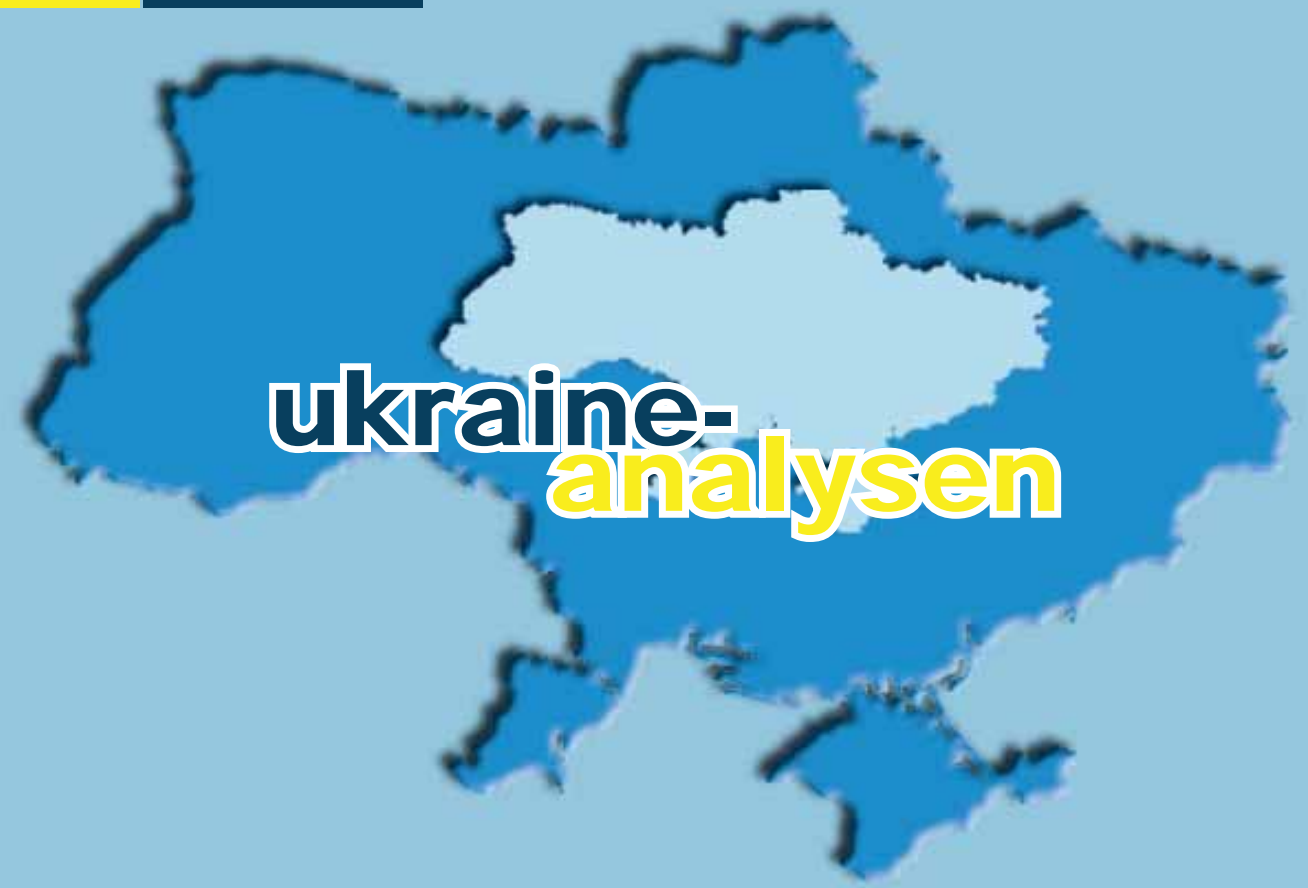

ukraine-analysen.de

\section{WOHNUNGSMARKT}

ANALYSE

Der ukrainische Wohnungssektor - schockabsorbierend und mobilitätseinschränkend.

Sebastian Klüsener, Freiburg

TABELLEN UND GRAFIKEN ZUM TEXT

Wohnungsmarkt und Bauwirtschaft

CHRONIK

Chronik vom 10. bis zum 24. Oktober 2006

Die nächsten Ukraine-Analysen erscheinen am 14. November 2006. Themen werden Politische Kultur und EU-Perspektiven sein.

\section{$\rightarrow$ Forschungsstelle Osteuropa}

\section{Deutsche Gesellschaft für} Osteuropakunde
Otto Wolff Stiftung 


\section{Analyse}

\section{Der ukrainische Wohnungssektor - schockabsorbierend und mobilitätseinschränkend}

Von Sebastian Klüsener, Institut für Kulturgeographie der Universität Freiburg

\section{Einleitung}

Der Wohnungsmarkt war in der Sowjetzeit stark reguliert. Art. 11 der sowjetischen Verfassung von 1977 schrieb vor, dass der Hauptanteil des Wohnraums in Städten in staatlichem Besitz sein musste, Grund und Boden waren komplett verstaatlicht. Im ländlichen Raum war allerdings auch unter der Sowjetherrschaft der überwiegende Teil der Häuser und Wohnungen in Privatbesitz. Bei der Errichtung von Wohnraum spielten staatliche Strukturen eine dominierende Rolle. Haushalte, die an einer Zuteilung interessiert waren, konnten sich auf Wartelisten eintragen. Die Mietpreise für staatliche Wohnungen waren aufgrund hoher Subventionen sehr niedrig und orientierten sich weder an der Nachfrage noch an den Kosten für Errichtung und Instandhaltung. Die sowjetische Gesetzgebung garantierte den Mietern lebenslanges Wohnrecht, eine Übertragung an die Nachkommen war einfach möglich.

$\mathrm{I}$

n den 1990er Jahren erfolgte eine Abkehr von dem hoch subventionierten und ineffizienten System hin zu einem mehr an marktwirtschaftlichen Gesichtspunkten orientierten Wohnungssektor, in dem private Akteure dominieren. Der Schwerpunkt der Reformen lag dabei auf der Privatisierung des öffentlichen Wohnraums und auf der Deregulierung des Immobilienmarktes.

Allerdings sieht Art. 47 der ukrainischen Verfassung analog zu Art. 44 der sowjetischen Verfassung von 1977 weiterhin vor, dass jeder ukrainische Bürger ein Recht auf Wohnraum hat. Dadurch ist der Staat prinzipiell verpflichtet, jeden Ukrainer in die Lage zu versetzen, Wohnraum bauen, kaufen oder mieten zu können. Sozial Bedürftigen wird garantiert, dass sie Wohnungen vom Staat bzw. den Städten und Gemeinden kostenlos oder zu einem bezahlbaren Preis zur Verfügung gestellt bekommen. In der Realität können diese Garantien aber oft nicht eingehalten werden.

\section{Privatisierung}

Tm Zuge der ab 1993 erfolgenden Privatisierung wer1 den Wohnungen und Häuser in der Regel kostenlos an die darin wohnenden Mieter übergeben. Von 1990 bis 2004 sank der Anteil des in öffentlicher Hand befindlichen Wohnraums in Städten von $72 \%$ auf $12 \%$. Im ländlichen Raum waren dagegen 1990 lediglich $12 \%$ des Wohnraums im Staatsbesitz gewesen, wobei der Anteil bis 2004 auf 2\% zurückging. (Siehe auch Grafik 2 und Tabelle 2 auf Seite 5) Der Privatisierungsprozess dauert bis heute an, auch wenn die Zahl der jährlich privatisierten Wohneinheiten seit Mitte der 1990er Jahre deutlich von ca. 900.000 auf unter 200.000 zurückgegangen ist. Am Ende soll nur noch ein kleiner Bestand an Sozialwohnungen in staatlicher Hand verbleiben.

Aufgrund des Privatisierungsprozesses kam dem Wohnungssektor die Rolle eines Schock-Absorbierers zu, da der Immobilienbesitz eine gewisse Stabilität und Absicherung gegenüber Notlagen bedeutet. Hiervon profitierten aber überwiegend Stadtbewohner, da Dorfbewohner in der Regel bereits vorher in privaten Häusern wohnten.

\section{Öffentlicher Wohnungsbau}

er öffentliche Wohnungsbau ist in der Transformationskrise aufgrund der Budgetschwierigkeiten vieler Städte und Gemeinden stark zurückgegangen, obwohl weiterhin ein hoher Bedarf besteht. Konnten im Jahr 1990 in der Ukraine noch 235.000 Familien bzw. Personen eine mit öffentlichen Mitteln errichtete Wohneinheit erhalten, waren es 2005 nur noch 20.000, wobei die Tendenz weiter fallend ist. Die Zahl der Wartenden, welche sich auf staatlichen Listen als wohnungsbedürftig eingetragen haben, ist dagegen lediglich von 2,6 Mio. auf 1,3 Mio. zurückgegangen. (Siehe auch Grafik 7 auf Seite 8)

Bei der Vergabe der mit staatlichen Geldern neu errichteten Wohnungen erhalten folgende Gruppen Priorität: Behinderte, Tschernobylopfer, Militärpersonal und Haushalte, welche in unakzeptablen Bedingungen mit weniger als $7 \mathrm{~m}^{2}$ Wohnraum pro Person leben. Ein niedriges Familieneinkommen ist dagegen kein Kriterium. Dass die Vorgaben nur wenig die Bedürftigkeit des Antragstellers berücksichtigen, wird auch daran deutlich, dass diejenigen Haushalte auf der Warteliste bei der Zuteilung bevorzugt werden sollen, welche bereit und in der Lage sind, eine neue durch die öffentliche Hand subventionierte 
Wohnung käuflich zu erwerben.

Während die Miethöhe im öffentlichen Wohnungssektor zu Sowjetzeiten auf nationaler Ebene festgelegt wurde, ist im Zuge der Transformation die Entscheidungskompetenz auf die lokale Ebene verlagert worden. Hierdurch kann nun vor Ort adäquater auf die jeweilige Lage reagiert werden, als dies vorher der Fall war. Die Mieten im öffentlichen Wohnungssektor sind weiterhin sehr niedrig, wozu auch ein detailliertes Discountsystem mit speziellen Vergünstigungen für bestimmte Gruppen beiträgt (Veteranen, Invalide, etc.). In der Regel reichen die Mieteinnahmen gerade zur Deckung der laufenden Kosten aus, weshalb Reparaturinvestitionen nur in akuten Fällen geleistet werden.

Bisher gibt es keinen Mechanismus, durch welchen Mieter aus öffentlichen Wohnungen ausziehen müssen, falls das Einkommen eine bestimmte Höhe übersteigt. Dies führt angesichts weitgehender Mieterrechte dazu, dass einmal vergebener öffentlicher Wohnraum praktisch in der Verfügungsgewalt des Mieters bzw. seiner Nachkommen verbleibt. Hierdurch gibt es im staatlichen Wohnungssektor praktisch keinen Leerstand.

\section{Soziale Schieflagen}

er starke Rückgang des öffentlichen Wohnungsbaus hat insbesondere für Dorfbewohner Konsequenzen, die in eine Stadt umziehen wollen. Bestanden in den 1980er Jahren noch gute Aussichten, innerhalb weniger Monate oder Jahre adäquaten Wohnraum von öffentlicher Seite zu erhalten, sind momentan die meisten darauf angewiesen, auf dem privaten Wohnungsmarkt zu suchen. Dies ist gerade in prosperierenden Städten schwierig, da die geforderten Mieten oft das Monatseinkommen einer Person übersteigen.

Eine weitere Bevölkerungsgruppe, die von der Entwicklung besonders betroffen ist, sind junge Erwachsene, die von zu Hause ausziehen und eine eigene Familie gründen wollen. Dass diese Gruppe es in der Transformation sehr schwierig hat, wurde vom ukrainischen Parlament bereits früh erkannt, wie entsprechende Gesetze zur Unterstützung junger Erwachsener aus der ersten Hälfte der 1990er Jahre verdeutlichen. In der Folge wurde aber zunächst nur wenig unternommen, um die gesetzlichen Vorgaben konkret umzusetzen. Erst 1998 beschloss das ukrainische Ministerkabinett, einen staatlichen Fond zur Unterstützung junger Ukrainer beim Wohnungsbau einzurichten, welcher Personen zwischen 18 und 30 Jahren subventionierte Kredite ausgibt. Allerdings konnte der staatliche Fond in den ersten Jahren seines Bestehens nur wenige Haushalte unterstützen, was auf eine Unterfinanzierung schließen lässt. Seit Ende 2004 sind die Ausschüttungen aber deutlich gesteigert worden.

Eine Analyse von Mukhna kommt jedoch zu der Schlussfolgerung, dass es sich bei dem Fond um eine Maßnahme handelt, welche die obere Mittelschicht und die Oberschicht beim Erwerb von Wohnraum unterstützt, da der geforderte Eigenanteil zu hoch ist. Diese Gruppen würden wahrscheinlich auch ohne diese Hilfe Zugang zu Wohnraum haben. Junge Familien mit geringen und mittleren Einkommen, die mit ihren Steuerzahlungen diesen Fond mitfinanzieren, haben dagegen keinen Zugang zu den subventionierten Krediten, was den Sinn dieses Wohnungsfonds in Frage stellt.

\section{Resümee}

Tnsgesamt lässt sich festhalten, dass es in den letzten 15 Jahren besonders in prosperierenden Städten zu einer Wohnraumknappheit und -verteuerung gekommen ist, wozu u.a. der starke Rückgang des öffentlichen Wohnungsbaus beigetragen hat. Die Problematik wird dadurch verschärft, dass sich sowohl die Vergabe öffentlich subventionierter Wohnungen als auch staatliche Unterstützungsmaßnahmen wie etwa der Fond zur Unterstützung junger Ukrainer beim Wohnungsbau nur durch eine geringe Bedürftigkeitsorientierung auszeichnen. Daher ist u.a. die Mobilität von Arbeitssuchenden, die in prosperierende Städte ziehen wollen, in der Ukraine zurzeit stark eingeschränkt. Dies hat auch negative Auswirkungen auf den Transformationsprozess, da sich ein Wechsel von Arbeitnehmern von nichtproduktiven zu produktiven Erwerbstätigkeiten deutlich erleichtert, wenn ein Wohnortwechsel einfach vollzogen werden kann.

Über den Autoren:

Sebastian Klüsener promoviert am Institut für Kulturgeographie der Universität Freiburg. Seine Dissertation befasst sich mit dem Wandel der Lebensgestaltungsmöglichkeiten und -strategien ukrainischer Dorfschulabgänger im Kontext von Globalisierung und postsowjetischer Transformation.

Lesetipp:

- Tsenkova, S. / Turner, B. (2004): The Future of Social Housing in Eastern Europe: Reforms in Latvia and Ukraine. In: European Journal of Housing Policy 4, S. $133-149$.

- Mukhna, A. (2004): Credit for Youth Housing. Konferenzbeitrag präsentiert auf der EERC/EROC Student Research Conference. Im Internet veröffentlicht unter: w w w.eerc.kiev.ua/ e ro c/ anconference/ a mukha.pdf 


\section{Tabellen und Grafiken zum Text}

\section{Wohnungsmarkt und Bauwirtschaft}

Grafik 1: Staatlicher Wohnungsbau 1980 - 2005 (in Tsd. Quadratmetern)

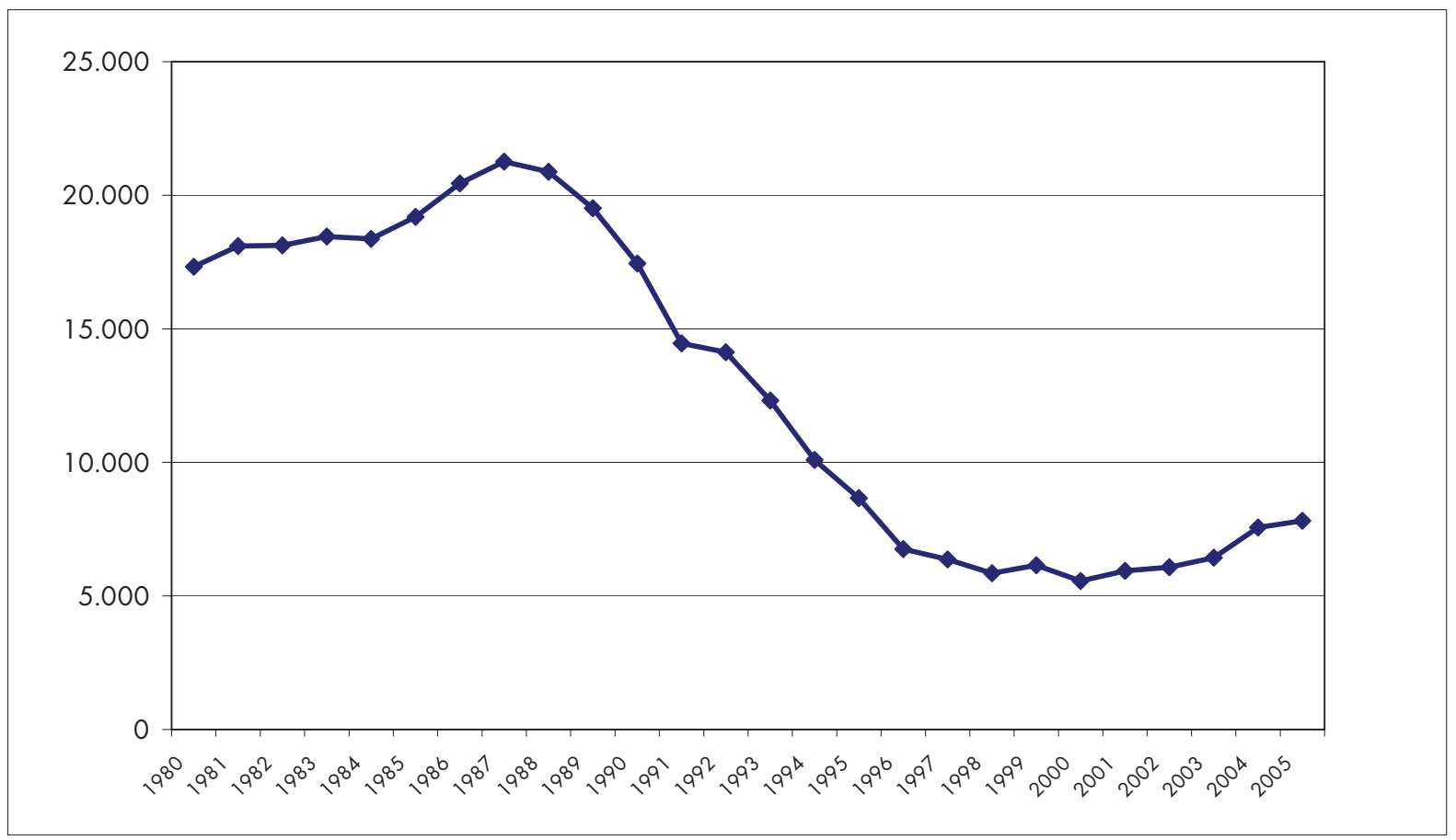

Quelle: Staatliches Statistikkomitee der Ukraine, http :/ / w w w.ukrsta t.gov.ua/ operativ/ operativ2005/ ibd/ ibd_rik/ ibd_u/ ezb_u.html

Tabelle 1: Staatlicher und privater Wohnungsbau 1985- 2004

\begin{tabular}{|c|c|c|c|c|}
\hline \multirow[b]{2}{*}{ Jahr } & \multicolumn{2}{|c|}{ Ukraine } & \multicolumn{2}{|c|}{ Stadt Kiew } \\
\hline & $\begin{array}{c}\text { staatlicher } \\
\text { Wohnungsbau (in } \\
\text { Tsd. } \mathrm{m}^{2} \text { ) }\end{array}$ & $\begin{array}{l}\text { privater Wohnungsbau } \\
\text { (in Tsd. } \mathrm{m}^{2} \text { ) }\end{array}$ & $\begin{array}{l}\text { kommunaler } \\
\text { Wohnungsbau } \\
\left(\text { in Tsd. } \mathrm{m}^{2}\right)\end{array}$ & $\begin{array}{c}\text { privater } \\
\text { Wohnungsbau } \\
\left(\text { in Tsd. } \mathrm{m}^{2}\right)\end{array}$ \\
\hline 1985 & 19.193 & 5.178 & 1.287 & 30 \\
\hline 1990 & 17.447 & 4.397 & 1.157 & 26 \\
\hline 1995 & 8.663 & 3.877 & 410 & 13 \\
\hline 2000 & 5.558 & 3.351 & 859 & 40 \\
\hline 2004 & 7.566 & 5.026 & 1.050 & 149 \\
\hline
\end{tabular}

Quelle: Statistisches Jahrbuch der Ukraine 2004, S. $240 f$. 
Grafik 2: Staatlicher und privater Anteil am gesamten Wohnungsbestand 1985 - 2004

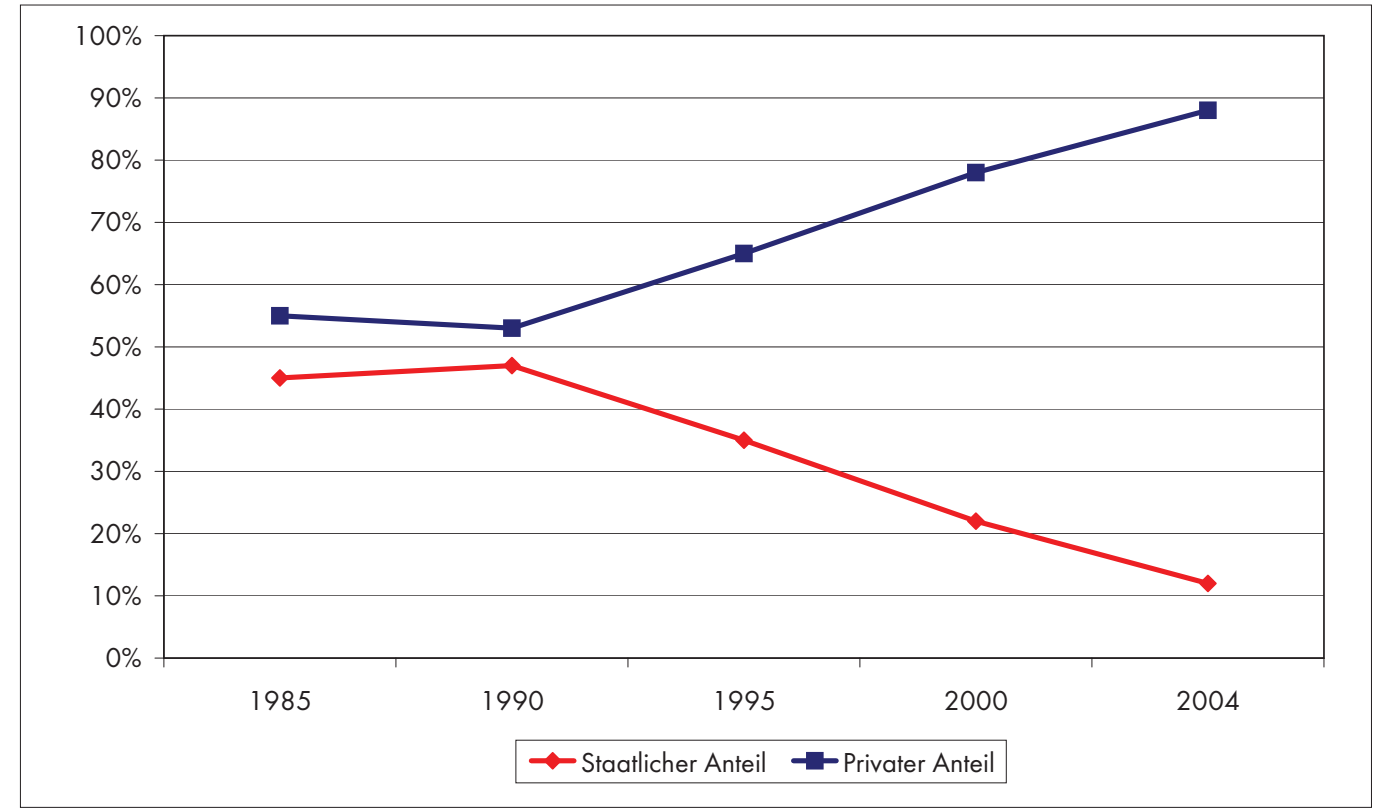

Quelle: Statistisches Jahrbuch der Ukraine 2004, S. 458

Tabelle 2: Staatlicher und privater Anteil am gesamten Wohnungsbestand (2004)

\begin{tabular}{|l|c|c|c|c|}
\hline & $\begin{array}{c}\text { Wohnfläche } \\
\text { insgesamt }\end{array}$ & Staatlicher Anteil & Privater Anteil & $\begin{array}{c}\text { Durchschnittswert pro } \\
\text { Kopf }\end{array}$ \\
\hline Ukraine insgesamt & 1.040 Mio. $\mathrm{m}^{2}$ & $12 \%$ & $88 \%$ & $21,8 \mathrm{~m}^{2}$ \\
\hline Städte & 665 Mio. $\mathrm{m}^{2}$ & $18 \%$ & $82 \%$ & $20,5 \mathrm{~m}^{2}$ \\
\hline Ländlicher Raum & 376 Mio. $\mathrm{m}^{2}$ & $2 \%$ & $98 \%$ & $24,4 \mathrm{~m}^{2}$ \\
\hline
\end{tabular}

Quelle: Statistisches Jahrbuch der Ukraine 2004, S. 458

Grafik 3: Neugeschaffener Wohnraum nach Regionen (in Quadratmetern pro 1.000 Personen)

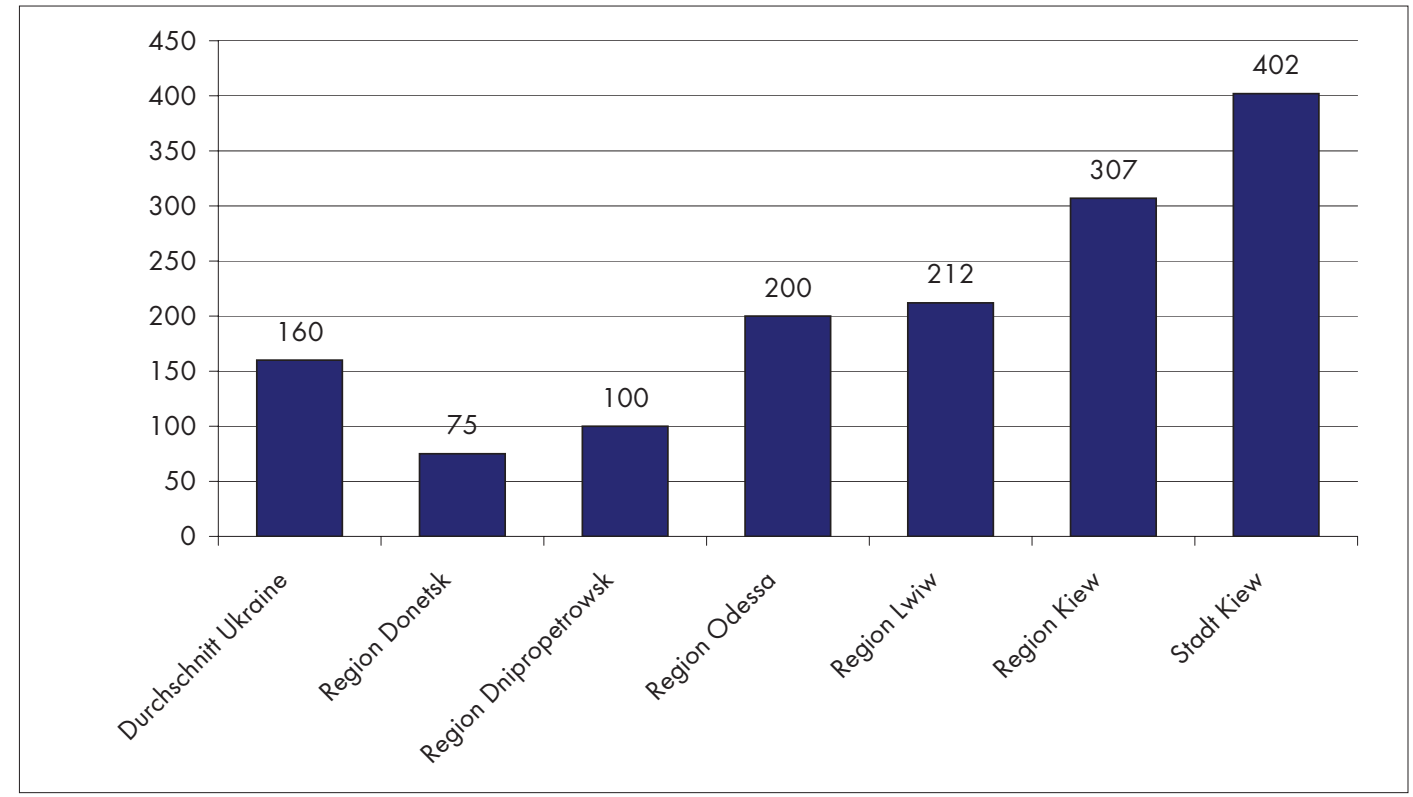

Quelle: Statistisches Jahrbuch der Ukraine 2004, S. 239. 
Tabelle 3: Neugebauter Wohnraum nach Regionen (in Quadratmetern pro 1.000 Personen)

\begin{tabular}{|c|c|}
\hline Region Donetsk & 75 \\
\hline Region Kirowograd & 96 \\
\hline Region Dnipropetrowsk & 100 \\
\hline Region Tscherkassi & 105 \\
\hline Region Luhansk & 106 \\
\hline Region Zaporissha & 107 \\
\hline Region Sumi & 112 \\
\hline Region Mikolajew & 117 \\
\hline Region Charkiw & 130 \\
\hline Region Cherson & 133 \\
\hline Regin Wolin & 133 \\
\hline Region Shitomir & 134 \\
\hline Krim & 138 \\
\hline Region Poltawa & 143 \\
\hline Region Zakarpatija & 150 \\
\hline Region Chmelnizki & 164 \\
\hline Region Riwne & 167 \\
\hline Region Ternopil & 173 \\
\hline Stadt Sewastopol & 185 \\
\hline Region Winizja & 198 \\
\hline Region Odessa & 200 \\
\hline Region Lwiw & 212 \\
\hline Region Tscherniwzi & 232 \\
\hline Region Iwano-Frankiwsk & 262 \\
\hline Region Kiew & 307 \\
\hline Stadt Kiew & 402 \\
\hline Durchschnitt Ukraine & 160 \\
\hline
\end{tabular}

Quelle: Statistisches Jahrbuch der Ukraine 2004, S. 239.

Grafik 4: Wohnungsbestand nach Größe (2005) (in Tsd. Wohnungen)

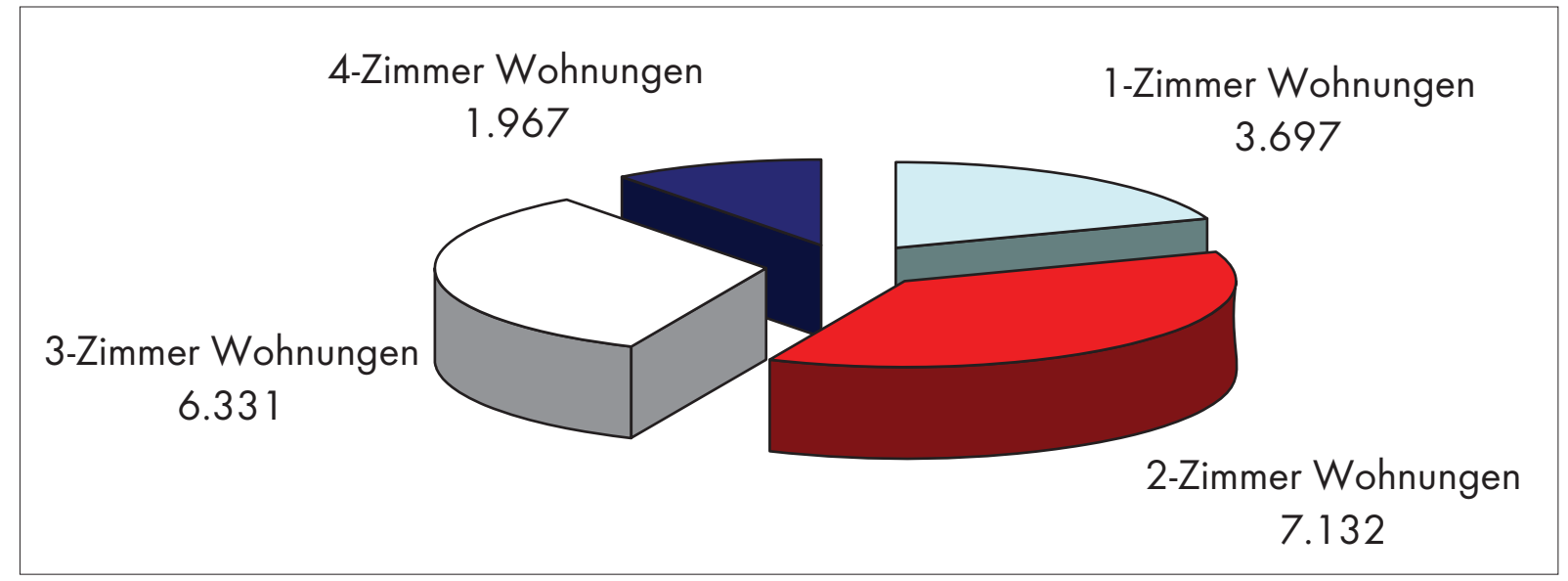

Quelle: Staatliches Statistikkomitee der Ukraine, http :/ / w w w.ukrsta tgov.ua/ operativ/ operativ2006 / zf/ zf_u/ $2005_{-}$u.htm 
Grafik 5: Durchschnittliche Wohnfläche pro Person $1990-2005\left(\mathrm{~m}^{2}\right.$ pro Person)

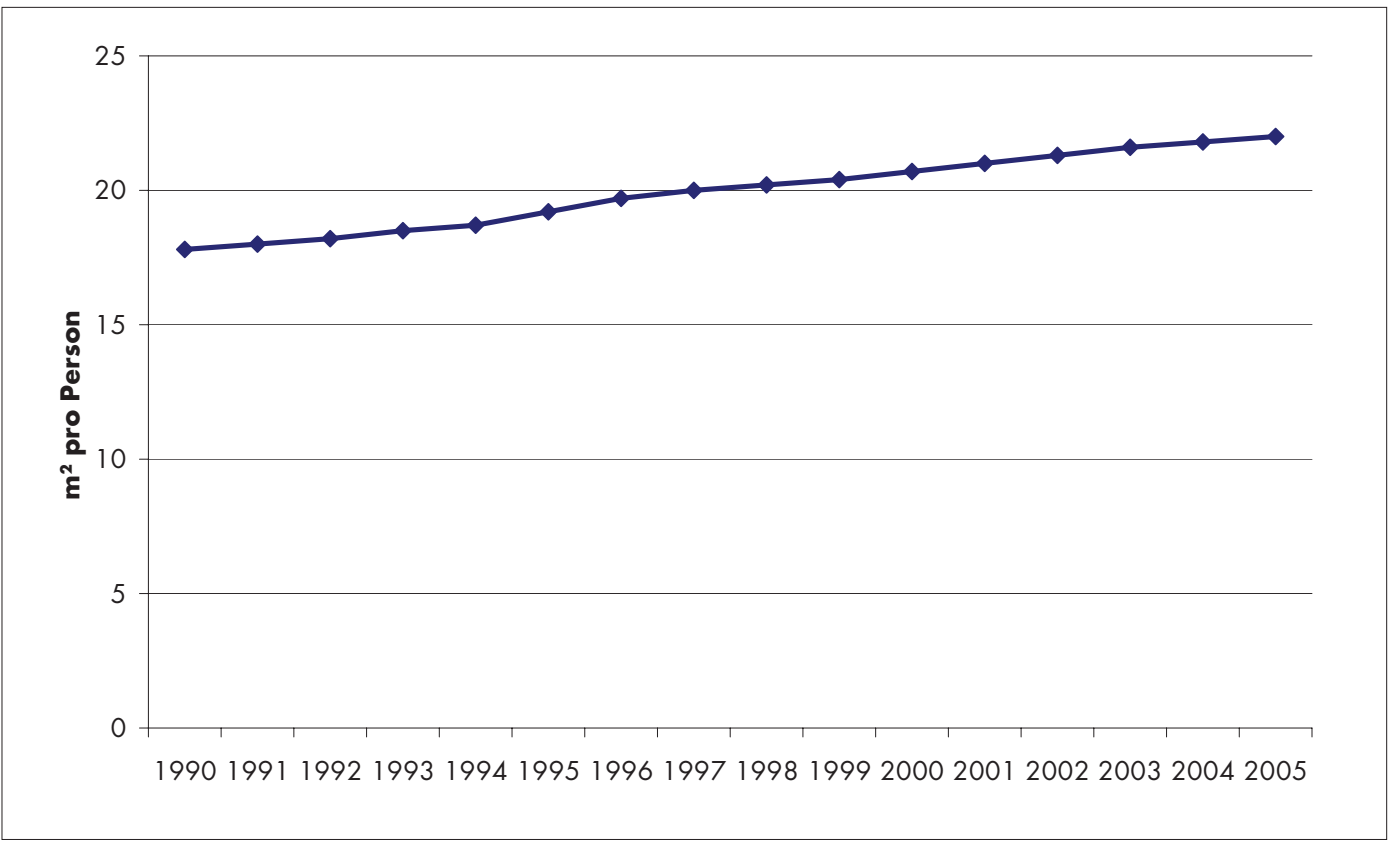

Quelle: Staatliches Statistikkomitee der Ukraine, http :/ / w w w.ukrsta t.g ov.ua/ operativ/ operativ2006/ zf/ zf_u/ 2005_u.htm

Grafik 6: Preisentwicklung von Eigentumswohnungen in Kiew 2000 - 2006 (US-Dollar pro $\mathrm{m}^{2}$ )

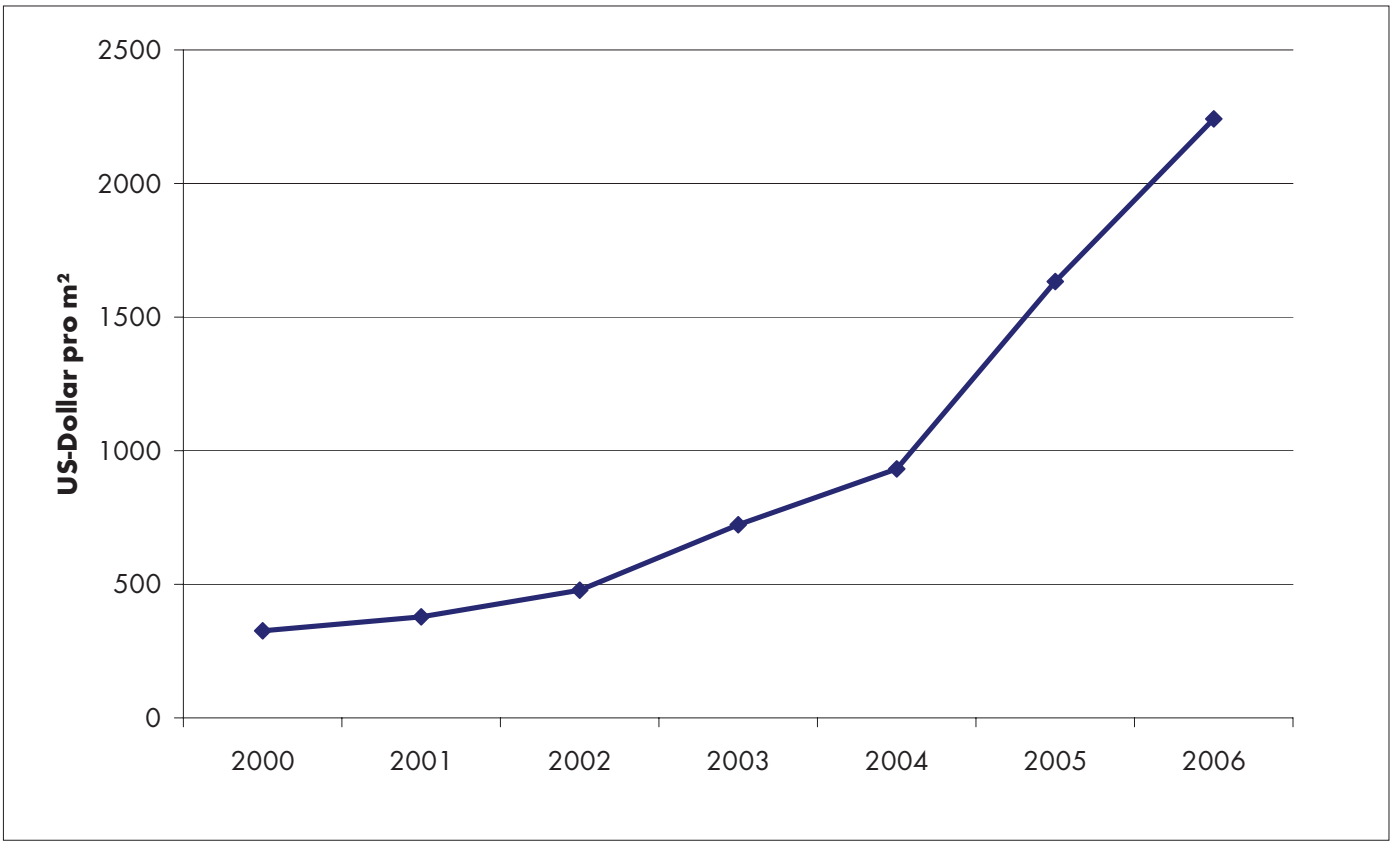

Anmerkung: Angegeben sind jeweils die Durchschnittspreise für 1- bis 3-Zimmer-Eigentumswohnungen im Monat September. Immobilienpreise werden in Kiew in der Regel in US-Dollar (als „Verrechnungseinheit") angegeben, so dass eine Umrechnung des Währungskurses nicht erfolgt.

Quelle: Immobilienagentur Blagovest, w w w.blagovestua 
Grafik 7: Vergabe von Sozialwohnungen in der Ukraine und in Kiew 1990 - 2004

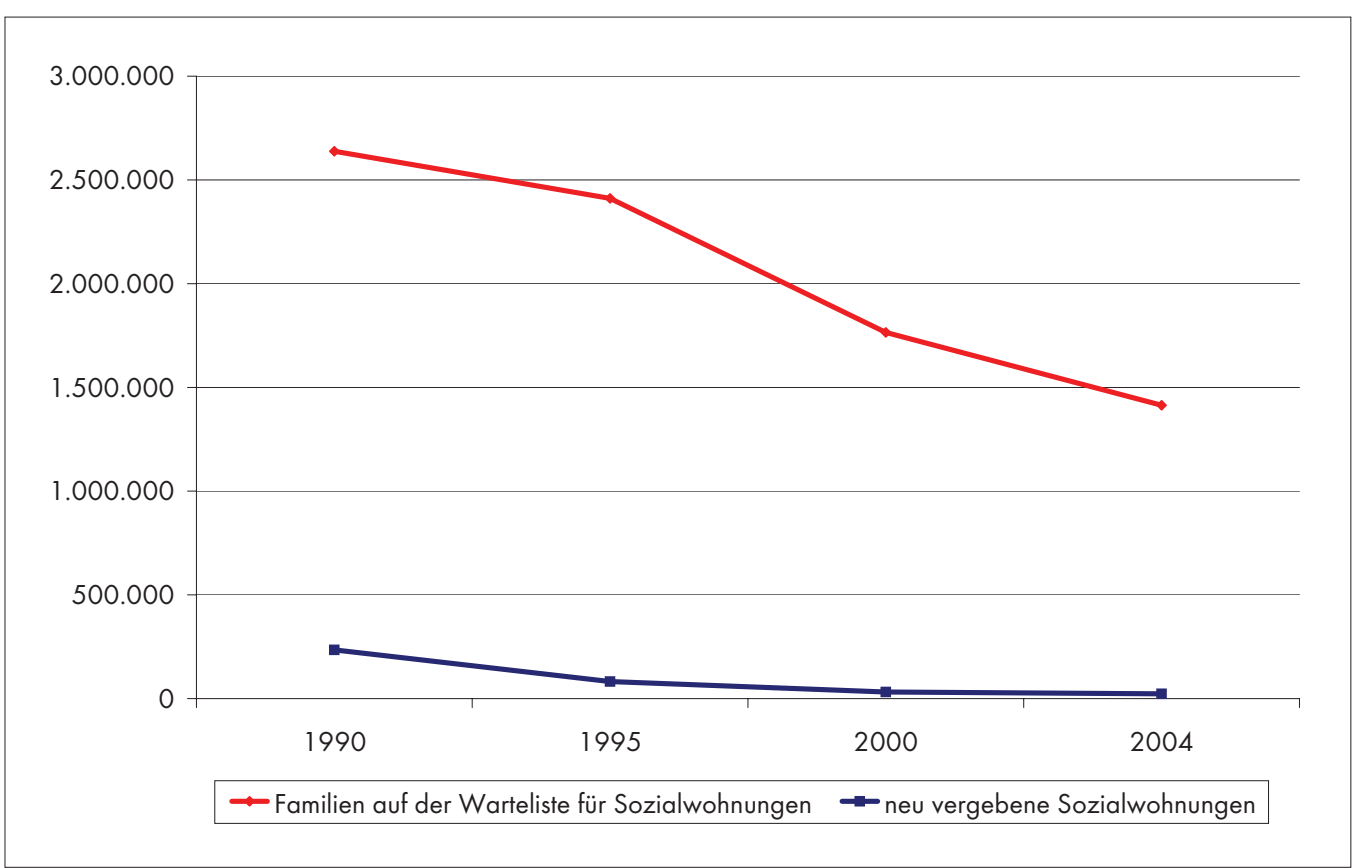

Tabelle 4: Vergabe von Sozialwohnungen in der Ukraine und in Kiew 1990 - 2004

\begin{tabular}{l|c|c|c|c|} 
& 1990 & 1995 & 2000 & 2004 \\
\hline Familien auf der Warteliste für Sozialwohnungen & 2.638 .000 & 2.411 .000 & 1.765 .000 & 1.414 .000 \\
Davon Kiew: & 230.000 & 251.000 & 229.000 & 193.000 \\
neu vergebene Sozialwohnungen & 235.000 & 82.000 & 32.000 & 23.000 \\
Davon: Kiew & 28.000 & 12.000 & 4.000 & 4.000
\end{tabular}

Quelle: Statistisches Jahrbuch der Ukraine 2004, S. 461; Statistisches Jahrbuch der Stadt Kiew 2005, S. 254

Tabelle 5 Staatliche Wohnungsbeihilfen in der Stadt Kiew (2004)

\begin{tabular}{l|c|c} 
& unterstützte Familien & Summe der Beihilfen (UAH) \\
Stadt Kiew & 94.624 & 4.570 .300 UAH (859.079 USD)
\end{tabular}

Quelle: Statistisches Jahrbuch der Stadt Kiew 2005, S. 245. 


\section{Chronik}

\section{Chronik vom 10. bis zum 24. Oktober 2006}

\begin{tabular}{|c|c|}
\hline 10.10 .2006 & $\begin{array}{l}\text { Präsident Viktor Juschtschenko entlässt Vladimir Gorbulin als Sekretär des Nationalen Sicherheitsrates und } \\
\text { ernennt Witalij Gaiduk zu seinem Nachfolger. }\end{array}$ \\
\hline 11.10 .2006 & $\begin{array}{l}\text { Präsident Viktor Juschtschenko und Ministerpräsident Viktor Janukowitsch erklären, dass sie noch Chancen auf } \\
\text { eine Einigung über eine Aufnahme der Partei Unsere Ukraine in die Regierungskoalition sehen. }\end{array}$ \\
\hline 11.10 .2006 & $\begin{array}{l}\text { Ukrainische Getreideexporteure protestieren gegen die Einführung von Beschränkungen des Getreideexports. } \\
\text { Der Weltmarktpreis für Getreide hat sich aufgrund des heißen Sommers gegenüber dem Vorjahr verdoppelt. } \\
\text { Die ukrainische Getreideernte dürfte dieses Jahr nach Schätzungen des Landwirtschaftsministeriums gegenüber } \\
\text { dem Vorjahr um knapp ein Viertel zurückgehen. Die ukrainische Regierung argumentiert, dass unter diesen } \\
\text { Bedingungen die Versorgung der eigenen Bevölkerung Vorzug habe. }\end{array}$ \\
\hline 12.10 .2006 & $\begin{array}{l}\text { Der Fraktionsvorsitzende der Partei Unsere Ukraine, Roman Bessmertny, erklärt, dass der Entschluss seiner Par- } \\
\text { tei in die Opposition zu gehen, „unumkehrbar“ sei. }\end{array}$ \\
\hline 14.10 .2006 & $\begin{array}{l}\text { Zum 64. Jahrestag der Gründung der ukrainischen nationalistischen Partisanenarmee UPA demonstrieren in } \\
\text { Kiew mehrere tausend Menschen für die Anerkennung der UPA Mitglieder als Veteranen des Zweiten Weltkrie- } \\
\text { ges. Dabei geht es zum einen um die Anerkennung der UPA als gleichberechtigt neben den Partisanen der sowje- } \\
\text { tischen Roten Armee und zum anderen für die UPA Veteranen um den Anspruch auf staatliche Vergünstigungen. } \\
\text { Die Polizei verhindert einen Zusammenstoß mit etwa tausend Gegendemonstranten, die von der Progressiven } \\
\text { Sozialistischen Partei organisiert wurden. Präsident Viktor Juschtschenko spricht sich für die Herstellung „his- } \\
\text { torischer Gerechtigkeit“ gegenüber den UPA Veteranen aus und unterschreibt einen entsprechenden Präsidial- } \\
\text { erlass, der allerdings nur symbolische Bedeutung hat, da die Entscheidungsbefugnis beim Parlament liegt. }\end{array}$ \\
\hline 17.10 .2006 & $\begin{array}{l}\text { Im Parlament erklärt der Fraktionsvorsitzende der Partei Unsere Ukraine, Roman Bessmertny, erneut den Wech- } \\
\text { sel seiner Partei in die Opposition und kündigt den Rücktritt der Parteimitglieder von ihren Regierungsposten } \\
\text { an. Er schlägt die Bildung einer umfassenden Opposition unter dem Titel Europäische Ukraine vor. Ministerprä- } \\
\text { sident Viktor Janukowitsch fordert alle Regierungsmitglieder auf, normal weiterzuarbeiten. }\end{array}$ \\
\hline 17.10 .2006 & $\begin{array}{l}\text { Das Parlament entscheidet die Anhörung zum ukrainischen WTO-Beitritt auf den 1. November zu verschie- } \\
\text { ben. }\end{array}$ \\
\hline 19.10 .2006 & $\begin{array}{l}\text { Die fünf Minister der Partei Unsere Ukraine, die von der Fraktion nominiert wurden, reichen ihren Rücktritt } \\
\text { ein. Die von Präsident Viktor Juschtschenko nominierten Außen- und Verteidigungsminister erklären, im Amt } \\
\text { bleiben zu wollen. Innenminister Juri Luzenko erklärt anschließend auf einer Pressekonferenz, dass er auf Bitte } \\
\text { von Juschtschenko sein Rücktrittsgesuch zurückgezogen habe. }\end{array}$ \\
\hline 23.10 .2006 & $\begin{array}{l}\text { Der ukrainische Sicherheitsrat erklärt, dass dem russischen kremlnahen politischen PR-Berater Gleb Pawlowskij } \\
\text { aufgrund von „Handlungen, die dem nationalen Interesse der Ukraine entsprechen“ für fünf Jahre die Einreise } \\
\text { in die Ukraine verboten wurde. Pawlowskij erklärt, dass er über das Verbot nicht informiert sei und noch am } \\
\text { Vortag für eine Tagesreise in der Ukraine gewesen sei. Probleme an der Grenze seien mit Hilfe des russischen } \\
\text { Konsulats gelöst worden. }\end{array}$ \\
\hline 24.10 .2006 & $\begin{array}{l}\text { Der russische Ministerpräsident Michail Fradkow trifft sich in Kiew mit seinem ukrainischen Amtskollegen } \\
\text { Viktor Janukowitsch. Auf der Sitzung der gemeinsamen Kommission für wirtschaftliche Zusammenarbeit wird } \\
\text { eine Bestandsaufnahme der Kooperation im Erdgasbereich bis zum Sommer } 2007 \text { beschlossen. Die Frage des } \\
\text { Preises für ukrainische Erdgasimporte soll hingegen nicht diskutiert werden. }\end{array}$ \\
\hline
\end{tabular}

Die Ukraine-Analysen werden mit Unterstützung durch die Otto-Wolff-Stiftung gemeinsam von der Forschungsstelle Osteuropa an der Universität Bremen und der Deutschen Gesellschaft für Osteuropakunde herausgegeben.

Die Meinungen, die in den Ukraine-Analysen geäußert werden, geben ausschließlich die Auffassung der Autoren wieder. Abdruck und sonstige publizistische Nutzung sind nach Rücksprache mit der Redaktion gestattet.

Redaktion und technische Gestaltung: Matthias Neumann, Heiko Pleines

Ukraine-Analysen-Layout: Cengiz Kibaroglu, Matthias Neumann

ISSN 1862-555X (c) 2006 by Forschungsstelle 0steuropa, Bremen

Forschungsstelle 0steuropa • Publikationsreferat • Klagenfurter Str. 3 • 28359 Bremen • Telefon: +49 421-218-7891 • Telefax: +49 421-218-3269

e-mail: publikationsreferat@osteuropa.uni-bremen.de • Internet-Adresse: www.ukraine-analysen.de 